BULLETIN OF THE

AMERICAN MATHEMATICAL SOCIETY

Volume 79, Number 4, July 1973

\title{
TRANSFORMATION GROUPS AND BIFURCATION AT MULTIPLE EIGENVALUES ${ }^{1}$
}

\author{
BY D. H. SATTINGER
}

Communicated by Hans F. Weinberger, January 12, 1973

1. Topological degree arguments show that bifurcation must take place at eigenvalues of odd multiplicity, while examples show bifurcation may not take place at eigenvalues of even multiplicity. The general problem of bifurcation at multiple eigenvalues is one which does not readily submit to a complete solution, so the approach must proceed by special cases. One important class for which a more detailed analysis is possible is that of problems invariant under a transformation group. The purpose of this note is to present some results in this direction. We would also like to call the reader's attention to the recent interesting work of D. Ruelle.

\section{Consider a nonlinear problem}

$$
\left(L_{0}+\lambda L_{1}\right) u+N(u)=0
$$

where $L_{0}, L_{1}$ and $N$ are continuous operators from a Banach space $X$ to $Y(X \subset Y)$. We assume that $N$ is Fréchet differentiable, that $N^{\prime}(0)=0$, and that $L_{0}+\lambda L_{1}$ is a Fredholm operator for all $\lambda$. (This means that the dimension of the null space of $L_{0}+\lambda L_{1}$ is equal to the codimension of its range in $Y$, and that its range is closed in $Y$.) Let $G$ be the group generated by $S^{1}$ (the unit circle with the usual addition) together with an inversion operator $i\left(i^{2}=e\right.$, where $e$ is the identity operator). Let $T_{g}$ be a representation of $G$ on $Y$. We label elements of $S^{1}$ by $\gamma, \delta$, where $0 \leqq \gamma$, $\delta<2 \pi$. Then $T_{\delta} T_{\gamma}=T_{\delta+\gamma}$. We assume that $L_{0}+\lambda L_{1}$ and $N$ commute with $T_{g}$ for all $g$ in $G$.

Let $\mathscr{N}_{\lambda}$ be the null space of $\left(L_{0}+\lambda L_{1}\right)$ and let $\left\{\lambda_{j}\right\}$ be the real numbers $\lambda$ for which $\mathscr{N}_{\lambda}$ is nontrivial. $\mathscr{N}_{\lambda_{j}}$ is invariant under $G$, and we assume $\mathscr{N}_{\lambda_{j}}$ is finite dimensional. If $\mathscr{N}_{\lambda_{j}}$ is irreducible, it is either one or two dimensional. Since bifurcation at simple eigenvalues is well understood (see [5] or [3]), let us consider the case $\operatorname{dim} \mathscr{N}_{\lambda_{j}}=2$. The eigenvalues of $T_{i}$ are \pm 1 , so in $\mathcal{N}_{\lambda_{j}}$ there is one vector $\varphi_{j}$ such that $T_{i} \varphi_{j}=\varphi_{j}$. Restricting (1) to the subspaces $X^{\prime}$ and $Y^{\prime}$ of all vectors $u$ such that $u=T_{i} u$, the corresponding null space $\mathcal{N}_{\lambda_{j}^{+}}^{+}$is one dimensional. (Since $N$ commutes with $T_{i}, T_{i} N(u)=N\left(T_{i} u\right)=N(u)$ if $u$ is in $X^{+}$.) We can now apply the usual bifurcation arguments to obtain a bifurcating curve of solutions

AMS (MOS) subject classifications (1970). Primary 35J60, $20 \mathrm{C} 35$.

Key words and phrases. Transformation groups, bifurcation theory.

${ }^{1}$ Partially supported by NSF GP-27973. 
$(\lambda, u)$ with $u$ in $X^{+}$. But then, applying the operators $T_{\delta}$, we get a one parameter sheet of solutions $\left(\lambda, T_{\delta} u\right), 0 \leqq \delta<2 \pi$, bifurcating from $\left(\lambda_{j}, 0\right)$.

TheOREM 1. Let $\mathscr{N}_{\lambda_{j}}$ be irreducible. If $\operatorname{dim} \mathscr{N}_{\lambda_{j}}=1$ there bifurcate two branches of solutions of (1) from $\left(\lambda_{j}, 0\right)$, all invariant under $S^{1}$. If $\operatorname{dim} \mathcal{N}_{\lambda_{j}}=2$ we get a one parameter sheet of solutions. Moreover, the solutions obtained above constitute the full set of solutions bifurcating from $\left(\lambda_{j}, 0\right)$.

The proof of the final statement proceeds along the following lines. Let $\left(\mu_{n}, u_{n}\right)$ be a sequence of solutions of $(1)$ tending to $\left(\lambda_{j}, 0\right)$. For large $n, u_{n}=a_{n} \varphi_{1}+b_{n} \varphi_{2}+O(1)$ where $\mathscr{N}_{\lambda_{j}}=\left[\varphi_{1}, \varphi_{2}\right]$. By applying an appropriate rotation $T_{\delta_{n}}$ we can put $b_{n}=0$. (This is a special property of the group $S^{1}$.) The problem is then reduced to an argument depending on the uniqueness of solutions obtained by an implicit function theorem argument. The details will be presented in a future paper.

The isotropy subgroup of a set $\mathcal{N}$ is the subgroup of transformations which leave $\mathcal{N}$ invariant. The subgroups of $S^{1}$ are the cyclic subgroups $\mathscr{C}_{n}$ generated by $T_{2 \pi / n}$ for some integer $n$. We continue to assume that $\mathcal{N}_{\lambda_{j}}$ is irreducible under $G$.

THEOREM 2. Let the isotropy subgroup of $\mathcal{N}_{\lambda_{j}}$ be $\mathscr{C}_{n_{j}} \cup i$. A sheet emanating from $\left(\lambda_{j}, 0\right)$ then consists of solutions invariant under $\mathscr{C}_{n_{j}} \cup i$ and this sheet must either tend to infinity or go to $\left(\lambda_{k}, 0\right)$, where $\mathscr{C}_{n_{j}} \subset \mathscr{C}_{n_{k}}$.

Proof. If (1) is restricted to the subspace $X_{j}$ of vectors invariant under $\mathscr{C}_{n_{j}}$ then we see only those eigenvalues $\lambda_{k}$ for which $\varphi_{k}$ belongs to $X_{j}$, thus $\mathscr{C}_{n_{j}} \subset \mathscr{C}_{n_{k}}$. We now restrict the subspace further to elements invariant under $i$. In that case $\lambda_{j}$ is simple, and topological degree arguments [2] show that the solutions bifurcating from $\left(\lambda_{j}, 0\right)$ must tend to infinity or another simple eigenvalue, in this case an eigenvalue $\lambda_{k}$ with $\mathscr{C}_{n_{j}} \subset \mathscr{C}_{n_{k}}$. We also see that $\operatorname{dim} \mathscr{N}_{\lambda_{k}} \cap X_{j}=2$.

3. Examples. The equation

$$
\Delta u+\lambda u+f(u)=0
$$

on a circular disk with $u=0$ on the boundary provides a simple illustration of the above ideas. We define $T_{\delta}$ by $\left(T_{\delta} u\right)(r, \theta)=u(r, \theta+\delta)$, and $T_{i} u(r, \theta)=u(r,-\theta)$. The null spaces $\mathscr{N}_{\lambda}$ are all irreducible $[4$, p. 484]. From each simple eigenvalue there bifurcate two branches of axisymmetric solutions, while from each double eigenvalue there bifurcates a one parameter sheet of solutions. The same results hold if $f=f(r, u)$.

As a second example, consider the Navier-Stokes equations

$$
\Delta u_{i}=\partial p / \partial x_{i}+u_{j} \partial u_{i} / \partial x_{j}, \quad \partial u_{i} / \partial x_{i}=0
$$

on a cylindrical domain $r_{1}<r<r_{2}$ (summation convention). The 
boundary conditions are periodic in the $z$ direction, and $u_{r}=u_{z}=0$, $u_{\theta}\left(r_{1}\right)=A, u_{\theta}\left(r_{2}\right)=B$ on the lateral surfaces. The appropriate group representation is $T_{\delta} \vec{u}(x)=O \vec{u}\left(O^{-1} x\right)$ where $O$ is a rotation about the $z$ axis through the angle $\delta$. For $T_{i}$ we take $T_{i} \vec{u}(x)=-\vec{u}(-x)$. An equation of the form (1) is obtained by linearizing about the special axisymmetric laminar solution (Couette flow, see [1]).

\section{REFERENCES}

1. K. Kirchgässner and P. Sorger, Stability analysis of branching solutions of the NavierStokes equations, Appl. Mech. Proc. XIIth Int. Cong. Appl. Mech., Springer, 1969.

2. P. H. Rabinowitz, Some global results for nonlinear eigenvalue problems, J. Functional Analysis 7 (1971), 487-512.

3. D. Sattinger, Topics in stability and bifurcation theory, Lecture Notes in Math., vol. 309, Springer-Verlag, Berlin and New York.

4. G. N. Watson, A treatise on the theory of Bessel functions, Cambridge Univ. Press, Cambridge; Macmillan, New York, 1944. MR 6, 64.

5. M. G. Crandall and P. H. Rabinowitz, Bifurcation from simple eigenvalues, J. Functional Analysis 8 (1971), 321-340. MR 44 \#5836.

6. D. Ruelle, Bifurcations in the presence of a symmetry group, I.H.E.S. preprint, Arch. Rational Mech. Anal. (to appear).

Department of Mathematics, University of California, Los Angeles, California 90024

Current address: School of Mathematics, University of Minnesota, Minneapolis, Minnesota 55416 